\title{
EQUIVALÊNCIA DE DULÇOR E PODER EDULCORANTE DE EDULCORANTES EM FUNÇÃO DA TEMPERATURA DE CONSUMO EM BEBIDAS PREPARADAS COM CHÁ-MATE EM PÓ SOLÚVEL ${ }^{1}$
}

\author{
Juliana Maria Porto CARDOSO², Juliana Rosa BATTOCHIO², Helena Maria André Bolini CARDELLO ${ }^{3, *}$
}

\begin{abstract}
RESUMO
No presente estudo foi verificado o efeito da temperatura de consumo na equivalência de doçura e no poder edulcorante de diferentes agentes adoçantes em bebida de chá-mate em pó solúvel. Foram avaliados: aspartame, sucralose, mistura ciclamato/sacarina 2:1, Stevia e acessulfame-K, tendo como referência a sacarose. Todos os estudos foram realizados a $6 \pm 2^{\circ} \mathrm{C}$ e a $45 \pm 2^{\circ} \mathrm{C}$. Primeiramente foi determinada a doçura ideal, utillizando-se escala do ideal com 30 provadores consumidores da bebida. Em seguida, foi determinada a doçura equivalente à sacarose (na doçura considerada ideal) para cada edulcorante estudado, e seu poder edulcorante nas duas temperaturas de estudo. Para tal foi aplicado o método de estimação de magnitude, utilizando-se uma equipe de 10 provadores selecionados e treinados. A doçura ideal de sacarose foi de $8,3 \%$ para a bebida de chá-mate solúvel, sem diferença significativa entre as temperaturas de estudo. Ocorreram diferenças sensoriais importantes em função da temperatura, pois, enquanto para alguns edulcorantes o aumento de temperatura provocou diminuição na potência edulcorante, para outros foi observado aumento do poder edulcorante. Portanto, não se deve generalizar as alterações no poder edulcorante em função da temperatura, pois ela pode variar em função da classe química envolvida e do meio de dispersão em que se encontra.

Palavras-chave: chá-mate; edulcorantes; poder edulcorante.
\end{abstract}

\section{SUMMARY}

EQUI-SWEETNESS AND SWEETENING POWER OF DIFFERENT SWEETENING AGENTS IN DIFFERENTS TEMPERATURES OF CONSUMPTION OF TEA DRINK IN SOLUBLE POWER. In this work the effect of temperature of consumption in the equi-sweetness and sweetening power of different sweetening agents was verified in tea drink in soluble powder. The panelists had evaluated: aspartame, sucralose, mixture of cyclamate/saccharin (2:1), Stevia [Stevia rebaudiana (Bert.) Bertoni] and acesulfame-K as sweeteners, having sucrose as reference. All sensory tests were carried-out at $6 \pm 2^{\circ} \mathrm{C}$ and $45 \pm 2{ }^{\circ} \mathrm{C}$. Firstly the ideal sweetness was determined by just-aboutright scale with 30 consumers of tea drink. After the ideal sweetness of sucrose determination, the equi-sweetness (in the same ideal sweetness) for each sweetening agent was determined, and its sweetening power in two temperatures of study, for such was applied the method of magnitude evaluation, with a panel of 10 selected and trained panelists. The ideal sweetness of sucrose concentration was of $8.3 \%$ for tea drink, without significant difference $(p \leq 0.05)$ between the temperatures of study. Important sensory differences occurred in function of temperature: some sweetening agent the increase of temperature promoted reduction in the sweetening power. For others, increase of the sweetening power was observed. Therefore, we can not generalize the alterations in the sweetening power in function of temperature, because it can vary in function of the involved chemical class and the way of dispersion where it is found.

Keywords: tea drink; sweeteners; sweetness potency.

\section{1 - INTRODUÇÃO}

Os indivíduos que, por diversas razões, precisam substituir a sacarose por adoçantes não calóricos procuram por produtos que sejam dotados de gosto e características próximas às da sacarose. Várias substâncias surgiram para suprir esta necessidade, mas poucas foram comprovadamente estabelecidas como seguras para o consumo humano, com bom poder edulcorante e estabilidade satisfatória.

A sacarina, o ciclamato, o aspartame, a sucralose e o extrato de folhas de Stevia, todos edulcorantes permitidos pela Legislação Brasileira [3] para utilização em alimentos e bebidas dietéticas com suas quantidades de ingestão diária aceitável definidas, possuem características sensoriais que podem diferir em função do produto em que se encontram e da temperatura de consumo.

\footnotetext{
1. Recebido para publicação em 15/08/2003. Aceito para publicação em 17/05/2004 (001190).

2. Departamento de Engenharia de Alimentos - Faculdade de Engenharia de Alimentos, Universidade Estadual de Campinas (UNICAMP).

3. Laboratório de Análise Sensorial - Faculdade de Engenharia de Alimentos, Universidade Estadual de Campinas (UNICAMP). Caixa Postal 6121, CEP: 13083-970. Campinas, SP Brasil.

* A quem a correspondência deve ser enviada.
}

É esta a razão pela qual não podemos generalizar um resultado sobre uma característica sensorial de um edulcorante obtido para um determinado produto. Por exemplo, um mesmo edulcorante que provoque a percepção de um gosto residual amargo intenso em um produto, pode ter esta característica atenuada em outro produto com outra estrutura química.

O mesmo pode acontecer em diferentes temperaturas.

Pesquisadores observaram um fato interessante na determinação do poder edulcorante do aspartame em relação à sacarose: com o aumento da concentração, ocorre diminuição do poder edulcorante [4, 5]. O mesmo fato ocorre com outros edulcorantes [4, 9].

Para que estes edulcorantes sejam aplicados com êxito é necessário que, além de sua segurança absoluta, eles apresentem características sensoriais agradáveis, com doçura semelhante à da sacarose. A única forma de se avaliar a aceitação de um edulcorante é pela análise sensorial.

Para que um adoçante possa substituir a sacarose, com êxito, em formulações de alimentos, é preciso realizar estudos que permitam o conhecimento prévio das concentrações dos adoçantes a serem utilizados e suas doçuras equivalentes em sacarose. As metodologias para 
a obtenção destas informações variam, podendo ser através do limiar absoluto [7], comparação pareada [18], variações do método do estímulo constante [6], e o método muito aplicado de estimação da magnitude e representação gráfica dos resultados normalizados, através da Lei de Stevens ou "power function" [12, 15].

TUNALEY, THOMSON \& McEWAN [16] utilizaram o método de escala de intensidade para avaliar a doçura de nove diferentes adoçantes, comparando-os a um padrão de glicose em solução a 5\%. Concluíram que houve muita variação nos dados obtidos, em relação aos provadores. Os autores concluíram que esta variação é dependente do tipo do adoçante, pois quanto mais complexa a percepção total, maior foi a variação nos resultados.

No método de estimação de magnitude, descrito por STONE \& OLIVER [15], os provadores devem receber uma amostra referência com uma intensidade designada com um valor arbitrário, por exemplo 10, seguida por uma série de amostras em ordem casualizada, com intensidades maiores ou menores que a referência. Os provadores deverão estimar o poder edulcorante das amostras desconhecidas e atribuir notas a elas, em relação à referência. Por exemplo, se a amostra tiver o dobro do poder edulcorante da amostra referência, deverá ter valor 20 , se for a metade 5 e, assim por diante. Simplesmente não se pode atribuir valor zero a qualquer amostra.

Os provadores são informados que a referência poderá ser reavaliada periodicamente, conforme a solicitação [11].

De acordo com MOSKOWITZ [12], o princípio da estimação de magnitude, ou função de potência, proporciona a obtenção de diversas ferramentas importantes para a avaliação de alimentos através da análise sensorial. Este autor cita uma analogia fisica dizendo que, no intervalo entre as temperaturas de $40^{\circ} \mathrm{C}$ e $80^{\circ} \mathrm{C}$ existem 40 graus de temperatura. Entretanto, $80^{\circ} \mathrm{C}$ não é percebido sensorialmente como tendo o dobro do aquecimento de $40^{\circ} \mathrm{C}$. Similarmente, o dobro de uma determinada concentração de sacarose ou de cloreto de sódio, não vai produzir a sua percepção em dobro, precisando quantificar estas medidas da forma como ela é percebida sensorialmente.

Os valores obtidos dos resultados dos provadores e os valores das concentrações avaliadas são normalizados, calculados os logaritmos desses resultados e colocados em um gráfico em coordenadas logaritmicas. Para cada adoçante (ou outro composto) é obtida uma reta, a qual obedece à lei de Stevens, ou "power function": $\mathrm{S}=\mathrm{aC}^{\mathrm{n}}$, onde $\mathbf{S}$ é o estímulo percebido, $\mathbf{C}$ é a concentração do estímulo, a é antilog do valor de $\mathrm{Y}$ no intercepto, e $\mathbf{n}$ é o coeficiente angular da reta. Regiões das retas dos adoçantes em que estão em mesmo nível, paralelo ao eixo da abcissa, possuem poder edulcorante equivalentes (MOSKOWITZ, 1970).

$\mathrm{O}$ aumento da temperatura geralmente causa o enfraquecimento, ou até mesmo a quebra das pontes de hidrogênio intramolecular nos açúcares, as quais afetam a conformação dos estímulos doces, alterando- os [13]. Por outro lado, mudanças na temperatura da camada vicinal de água (ou saliva) contribuem para uma modificação da membrana em si, podendo levar a uma alteração da mensagem. Uma vez que a tradução do estímulo químico, em uma mensagem nervosa, se dá através de um número de etapas, a temperatura pode influenciar cada uma delas [1].

BIRCH, LATYMER \& HOLOWAY [2] observaram que a intensidade da doçura de açúcares e adoçantes sintéticos aumenta quando se aumenta a temperatura, enquanto que a persistência diminui para os açúcares, mas não para outros adoçantes.

De acordo com GREEN \& FRANKMANN [8], é mais fácil predizer o comportamento dos açúcares quando a temperatura é alterada, do que o comportamento dos adoçantes artificiais.

Entre os métodos sensoriais existentes para se medir a quantidade ideal de um determinado componente, a ser adicionado para provocar a melhor aceitação e preferência de um grupo de julgadores. A a escala-do-ideal é o método afetivo mais aplicado, tanto devido à confiabilidade e validade de seus resultados como à simplicidade em ser utilizada pela equipe. Nesta análise, a equipe de provadores avalia as amostras e registra suas respostas em escala específica, o quão ideal estas amostras encontram-se, em relação ao atributo que se deseja avaliar (por exemplo doçura, textura e outros), conforme o método de VICKERS [17].

Os dados obtidos são então submetidos à análise estatística através de gráfico de distribuição das respostas sensoriais (em porcentagem), em função da concentração do componente que está variando e, também, por regressão linear simples entre os valores hedônicos e a concentração do componente que está variando.

Com a aplicação da análise de aceitação, com a escala-do-ideal, é possível transformar dados subjetivos em objetivos e obter informações importantes sobre a concentração adequada de um composto a ser adicionado em um alimento ou bebida.

Por outro lado, a preocupação com a saúde impulsionou a pesquisa e o desenvolvimento de alimentos com baixa caloria e adoçantes não calóricos.

As folhas de erva-mate torradas são comercializadas para a obtenção de uma bebida estimulante, em função da presença de cafeína e pequenas quantidades de teobromina, teofilina e xantina [14].

A bebida de chá-mate é amplamente conhecida e consumida por diferentes faixas etárias e socioeconômicas. Pode ser consumida quente, gelada ou a temperatura ambiente. Além disso, é consumida adoçada com sacarose ou adoçante de mesa. Em função de sua popularidade, será utilizada como base no presente estudo.

O objetivo do presente estudo foi determinar a doçura ideal para bebida de chá-mate em pó solúvel, adoçada com sacarose, e determinar a quantidade equivalente de diferentes edulcorantes para promoverem a mesma doçura, e também determinar o poder edulcorante em relação à sacarose. 


\section{2 - MATERIAL E MÉTODOS}

\section{1 - Material}

Foi utilizado chá-mate em pó solúvel da marca comercial Leão Júnior (fabricante), preparado conforme as instruções do fabricante.

O chá-mate assim preparado foi adoçado com cinco diferentes edulcorantes: Aspartame puro (Nutrasweet); Extrato de folhas de Stevia (Steviafarma do Brasil); Mistura ciclamato / sacarina na proporção de 2:1; Sucralose (Splenda, Johnson), Sacarose (Sigma Chemical Co.) e Acessulfame-K (Slim diet).

\subsection{1 - Preparação das amostras}

As amostras de chá-mate foram preparadas dissolvendo-se 4,6g do pó solúvel em $1000 \mathrm{~mL}$ de água mineral, conforme as instruções do fabricante, tendo sido adoçadas com os diferentes edulcorantes em concentrações pré-definidas, cerca de uma hora de antecedência. Estas foram aquecidas em banho-maria até atingirem a temperatura de $45^{\circ} \mathrm{C} \pm 2^{\circ} \mathrm{C}$ e, em seguida, colocadas em garrafas térmicas. As amostras de chámate quente foram então, servidas aos provadores para serem avaliadas sensorialmente. As avaliações sensoriais foram realizadas em cabines individuais e as amostras codificadas com algarismos de três dígitos, em copos plásticos brancos descartáveis.

\section{2 - Análise sensorial}

\subsection{1 - Determinação da concentração ideal de sacarose}

A determinação da concentração ideal de sacarose (\%) a ser adicionada ao chá-mate quente foi realizada através de um teste de aceitação, utilizando escala do ideal [10].

Foram utilizados 30 provadores não treinados, porém consumidores de chá-mate. As amostras foram apresentadas de forma monádica, sendo testadas as concentrações de 5,$0 ; 7,5 ; 10,0 ; 12,5$ e $15 \%$ de sacarose.

\subsection{2 - Pré-seleção da equipe de provadores}

A pré-seleção dos provadores foi realizada através de testes triangulares, aplicados à análise seqüencial [11]. Para os testes, foram utilizadas duas amostras de chá-mate quente, adoçadas com sacarose, que apresentavam diferença significativa entre si ao nível de $1 \%$. Para determinar a diferença de concentração entre estas duas amostras foi realizado um teste pareado com 20 provadores.

$\mathrm{Na}$ análise seqüencial foram utilizados os valores para $\mathrm{p}=0,45$ (máxima inabilidade aceitável), $\mathrm{p} 1=0,70$ (mínima habilidade aceitável), e para os riscos $\alpha=0,05$ (probabilidade de aceitar um candidato sem acuidade sensorial) e $\beta=0,05$ (probabilidade de rejeitar um candidato com acuidade sensorial).

\subsection{3 - Determinação da equivalência de doçu- ra}

A medida do poder edulcorante relativo dos edulcorantes e da mistura de edulcorantes foi realizada de acordo com o método de estimação de magnitude [11], o qual proporciona a medida quantitativa direta da intensidade subjetiva de doçura.

As amostras foram apresentadas aos provadores através de blocos completos casualizados, acompanhadas de uma referência de chá-mate adoçado com sacarose na concentração de $8,3 \%$ (concentração ideal de sacarose a ser adicionada ao chá-mate, determinada no teste de aceitação com escala do ideal para sacarose).

Foram utilizados dez provadores selecionados em função do poder discriminativo através de análise seqüencial. Estes provadores foram treinados para utilizarem escalas de magnitude com padrões de intensidades de doçura diferentes.

Para as avaliações de cada edulcorante em chá-mate (gelado e quente) foram utilizadas as séries de concentrações apresentadas na Tabela 1, com um fator de multiplicação de 1,6.

Neste método, os provadores selecionados e treinados receberam uma amostra referência, com intensidade designada por um valor arbitrário de doçura 100 , seguida de várias amostras codificadas e casualizadas, com intensidades maiores ou menores que a referência (conforme indicado na Tabela 1). Solicitou-se aos provadores que estimassem as intensidades de doçura das amostras codificadas em relação à referência. Por exemplo, se a amostra apresentasse o dobro da doçura da referência, deveria receber valor 200 , se apresentasse a metade da doçura, 50 e, assim por diante.

TABELA 1. Concentrações de sacarose (referência), aspartame, Stevia, ciclamato/sacarina $2: 1$, sucralose e acessulfame- $\mathrm{K}$ avaliadas para determinar a concentração equivalente à sacarose a $8,3 \%$, em chá-mate a $6^{\circ} \mathrm{C}$ e a $45^{\circ} \mathrm{C}$.

\begin{tabular}{|c|c|c|c|c|c|}
\hline \multirow{2}{*}{$\begin{array}{c}\text { Estímulo } \\
\text { Sacarose }\end{array}$} & \multicolumn{5}{|c|}{$\begin{array}{c}\text { Concentrações para determinação da } \\
\text { equivalência a } 8,3 \% \text { de sacarose }\end{array}$} \\
\hline & 3,1250 & 5,0000 & 8,300 & 12,8000 & 20,4800 \\
\hline Aspartame & 0,0215 & 0,0344 & 0,0550 & 0,0880 & 0,1408 \\
\hline Ciclamato/sacarina & 0,0141 & 0,0225 & 0,0360 & 0,0576 & 0,0922 \\
\hline Stevia & 0,0391 & 0,0625 & 0,1000 & 0,1600 & 0,2560 \\
\hline Sucralose & 0,0063 & 0,0100 & 0,01600 & 0,0256 & 0,0410 \\
\hline Acessulfame $\mathrm{K}$ & 0,0200 & 0,0340 & 0,0550 & 0,0880 & 0,1408 \\
\hline
\end{tabular}

Para a análise dos dados, os valores de magnitude de doçura estimados foram convertidos para valores logarítmicos e expressos utilizando média geométrica. As curvas para concentração versus resposta sensorial, para cada edulcorante, foram correspondentes a uma função de potência ("power function") com a se- 
guinte característica: $\mathbf{S}=\boldsymbol{a} \boldsymbol{C}^{\boldsymbol{n}}$, onde $\mathbf{S}$ é a sensação percebida, $\mathbf{C}$ é a concentração do estímulo, a é o antilog do valor de y no intercepto e $\mathbf{n}$ é o coeficiente angular da reta obtida [12].

\section{3 - RESULTADOS E DISCUSSÃO}

A opinião dos provadores, verificada no teste utilizando escala do ideal, foi transformada em dados numéricos $(-4 \mathrm{a}+4)$, sendo que a doçura ideal correspondia ao valor 0 (zero). A partir da equação da reta obtida, calculou-se que a concentração ideal de sacarose a ser adicionada à bebida de chá-mate gelada é de $8,3 \%$ e para o chá quente $8,15 \%$.

Foi realizado um teste discriminativo (triangular) para determinar se a diferença encontrada era estatisticamente diferente, e o resultado encontrado foi que a diferença sensorial entre as duas concentrações, tanto a $6^{\circ} \mathrm{C}$ quanto a $45^{\circ} \mathrm{C}$ não era estatisticamente significativa $(\mathrm{p} \leq 0,01)$.

Portanto, a doçura ideal tanto para o chá quente como para o chá frio foi considerada de $8,3 \%$ de sacarose.

A partir da concentração de sacarose, para provocar a doçura ideal do chá-mate tanto gelado como quente, foi utilizada a metodologia estimação de magnitude.

TABELA 2. Coeficiente angular, intercepto na ordenada, coeficiente de correlação linear e função de potência ("power function") dos resultados obtidos nos testes utilizando escala de magnitude, para determinar as equivalências de doçura do aspartame, mistura ciclamato/sacarina $2: 1$, Stevia, sucralose e acessulfame $\mathrm{K}$ em relação à concentração de $8,3 \%$ de sacarose em chá-mate gelado $\left(6^{\circ} \mathrm{C}\right)$ e quente $\left(45^{\circ} \mathrm{C}\right)$.

\begin{tabular}{|c|c|c|c|c|}
\hline \multicolumn{5}{|c|}{ Chá a $45^{\circ} \mathrm{C}$} \\
\hline Edulcoranto & Coeficiente & Intercepto & & Função de Potência \\
\hline Lunturiante & Angular (b) & na Ordenada (a) & $\pi$ & (“Power Function”) \\
\hline Sacarose & 1,79 & $-1,77$ & 0,95 & $S=0,017 . C^{1,79}$ \\
\hline Aspartame & 1,51 & 1,91 & 0,99 & $S=81,28 . C^{1,51}$ \\
\hline Ciclam/sac & 1,47 & 2,12 & 0,97 & $S=131,82 \cdot C^{1,47}$ \\
\hline Stevia & 1,33 & 1,33 & 0,98 & $S=21,38 . C^{1,33}$ \\
\hline Sucralose & 1,87 & 3,36 & 0,99 & $S=2290,87 \cdot C^{1,87}$ \\
\hline Acessulfame-K & * & * & * & * \\
\hline \multicolumn{5}{|c|}{ Chá a $6^{\circ} \mathrm{C}$} \\
\hline \multirow{2}{*}{ Edulcorante } & Coeficiente & Intercepto & \multirow{2}{*}{$\mathrm{R}$} & Função de Potência \\
\hline & Angular (b) & na Ordenada (a) & & (“Power Function”) \\
\hline Sacarose & 2,07 & $-2,07$ & 0,93 & $S=0,0085 . C^{2,07}$ \\
\hline Aspartame & 1,68 & 2,13 & 0,93 & $S=134,89 \cdot C^{1,68}$ \\
\hline Ciclam/sac & 1,61 & 2,21 & 0,99 & $S=162,18 \cdot C^{1,61}$ \\
\hline Stevia & 1,31 & 1,22 & 0,98 & $S=16,60 \cdot C^{1,31}$ \\
\hline Sucralose & 0,91 & 1,45 & 0,90 & $S=28,18 . C^{0,91}$ \\
\hline Acessulfame-K & 1,95 & 2,47 & 0,99 & $S=295,12 \cdot C^{1,95}$ \\
\hline
\end{tabular}

*Não foi possivel determinar os valores para o Acessulfame-K pois a doçura não foi percebida a $45^{\circ} \mathrm{C}$.

A partir dos resultados encontrados na Tabela 2, foi possivel calcular a quantidade equivalente das substâncias adoçantes avaliadas para proporcionar a mes- ma doçura que a sacarose a $8,3 \%$, em chá nas duas temperaturas de estudo. Tais quantidades encontramse na Tabela 3.

Com esses valores foi calculado o poder edulcorante das substâncias, as quais encontram-se na Tabela 4.

O poder edulcorante é definido como o número de vezes que uma substância é mais doce que a sacarose.

Na Figura 1 é possivel diferenciar de forma clara as alterações que ocorreram com os edulcorantes em função da temperatura.

TABELA 3. Concentração de aspartame, mistura ciclamato/ sacarina 2:1, Stevia, sucralose e acessulfame-K que equivale à concentração de $8,3 \%$ de sacarose em bebida de chá-mate quente e gelada.

\begin{tabular}{ccc}
\hline Edulcorante & $\begin{array}{c}\text { Concentração equivalente a } \\
8,3 \% \text { de sacarose em bebida } \\
\text { de chá-mate a } 6^{\circ} \mathrm{C}(\%)\end{array}$ & $\begin{array}{c}\text { Concentração equivalente a } \\
8,3 \% \text { de sacarose em bebida } \\
\text { de chá-mate a } 45^{\circ} \mathrm{C}(\%)\end{array}$ \\
\hline Aspartame & 0,030 & 0,050 \\
Ciclamato/sacarina & 0,025 & 0,030 \\
Stevia & 0,100 & 0,070 \\
Sucralose & 0,015 & 0,012 \\
Acessulfame-K & 0,030 & $\mathrm{NC}$ \\
\hline
\end{tabular}

*Não foi possivel calcular a concentração equivalente a $8,3 \%$ de sacarose para o edulcorante acessulfame $\mathrm{K}$, pois ocorreu inversão da curva.

TABELA 4. Poder edulcorante (número de vezes que o composto é mais doce que a sacarose na concentração de $8,3 \%$ em chá-mate quente e gelado).

\begin{tabular}{ccc}
\hline Edulcorante & Chá-m ate a $6^{\circ} \mathrm{C}$ & $\mathrm{Chá}-\mathrm{m}$ ate a $45^{\circ} \mathrm{C}$ \\
\hline Aspartame & 277 & 163 \\
C ic la ma to/sacarina & 332,4 & 272 \\
S tevia & 83,1 & 116 \\
Sucralose & 554 & 679 \\
A cessulfame-K & 277 & -
\end{tabular}

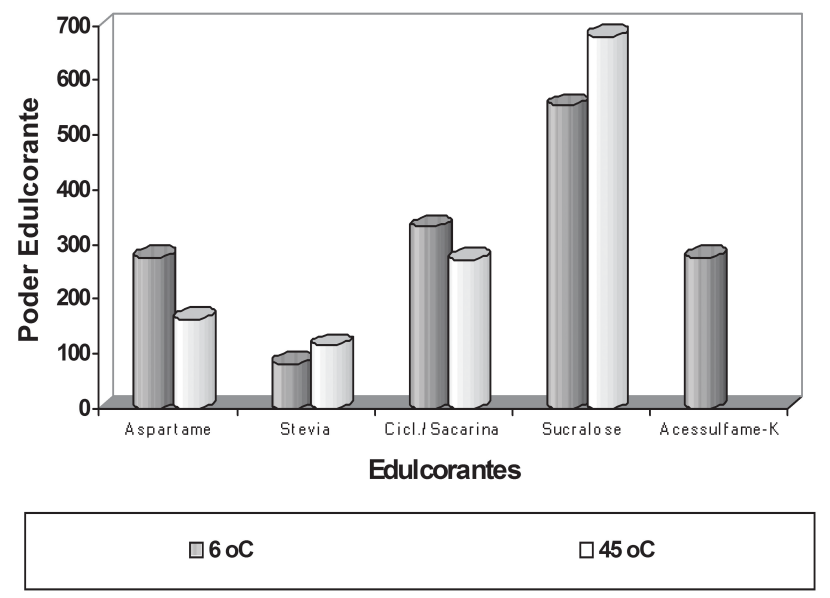

FIGURA 1. Poder edulcorante ( $\mathrm{n}$ - de vezes mais doce que a sacarose) das substâncias avaliadas em chá-mate gelado $\left(6^{\circ} \mathrm{C}\right)$ e quente $\left(45^{\circ} \mathrm{C}\right)$.

Os resultados encontrados são bastante importantes, pois, enquanto para o aspartame e para a mistura 
ciclamato/sacarina 2:1 o aumento de temperatura provocou diminuição na potência edulcorante, para a Stevia e para a sucralose foi observado aumento do poder edulcorante.

Esses resultados comprovam que a percepção dos gostos das substâncias depende de vários fatores, como estes confirmados: do meio de dispersão em que se encontram e da temperatura, dependendo da classe química.

Por exemplo, para o aspartame, que é um dipeptídeo, e para a mistura de ciclamato e sacarina, ácido ciclohexilsulfâmico e imida do ácido sulfobenzóico, a temperatura causou diminuição do poder edulcorante, enquanto para a Stevia que é um glicosídeo terpênico e, para a sucralose que é a sacarose clorada (mais especificamente 1,6 - dicloro- 1,6 - didesoxi - $\beta$-D-frutofuranosil-4-cloro4-desoxi- $\alpha$-D-galactopiranosídeo), a temperatura provocou aumento do poder edulcorante.

Portanto, não é possivel prever ou generalizar o comportamento de um agente edulcorante sem um estudo aprofundado das condições de seu consumo.

Em função dos estudos realizados, é possível sugerir que em rótulos de adoçantes de mesa comercializados, seria apropriado que as indicações de quantidades equivalentes neles contidas, fossem mais especificas para melhor orientar os consumidores.

\section{4 - CONCLUSÕES}

A partir dos resultados obtidos foi possível concluir que:

- A doçura ideal de sacarose foi de $8,3 \%$ para a bebida de chá-mate solúvel, sem diferença significativa entre as temperaturas de estudo;

- Ocorreram diferenças sensoriais importantes em função da temperatura, pois enquanto para o aspartame e para a mistura ciclamato/sacarina 2:1 o aumento de temperatura provocou diminuição no poder edulcorante, para a Stevia e para a sucralose foi observado aumento do poder edulcorante;

- Não se deve generalizar as alterações no poder edulcorante em função da temperatura, pois ela pode variar em função da classe química envolvida e do meio de dispersão em que se encontra.

\section{5 - REFERÊNCIAS BIBLIOGRÁFICAS}

[1] BARTOSHUK, L.M., RENERT, K., RODIN, J., STEVENS, C. Effects of temperature on the perceived sweetness of sucrose. Physiol. Behav., v.28, p. 905-1002, 1982.

[2] BIRCH, G.G., LATYMER, Z., HOLOWAY, M.0. Intensitytime relationships in sweetness- evidence for a queue hypothesis in taste chemoreception. Chem. Senses, v.5, p.63-79, 1980 .

[3] BRASIL. Ministério da Saúde. Secretaria de Vigilância Sanitária. Portaria no. 318 de 24 de novembro de 1995. Aprova o uso de Sucralose com a função de edulcoran- te em alimentos e bebidas dietéticas; Diário Oficial da República Federativa do Brasil, Brasilia, no. 227, p.194061, 28 nov. 1995.

[4] CARDELlO, H.M.A.B., DA SILVA, M.A.A.P., DAMÁSIO, M.H. Measurement of the relative sweetness of stevia extract, aspartame and cyclamate/saccharin blend as compared to sucrose at differents concentrations. Plant Foods for Human Nutrition, v.54 n.2, p. 119-130, 1999.

[5] CLONINGER, M.R., BALDWIN, R.E. L-aspartyl-Lphenylalanine methyl ester (aspartame) as a sweetener. J. Food Sci., v.39, p.347-9, 1974.

[6] DE GRAAF, C., FRIJTERS, J.E.R. A psychophysical investigation of Beidler's mixture equation. Chem. Senses, $v$. 11, p. 295-314, 1986.

[7] FABIAN, F.W., BLUM, H. B. Relative taste potency of some basic food constituents and their competitive and compensatory action. Food Res., v. 8, p. 179-93, 1943. Apud TUNAlEY, A., THOMSON, D.M.H., McEWAN, J.A. Determination of equi-sweet concentrations of nine sweeteners using a relative rating technique. Int. J. Food Sci. Technol., v. 22, p. 627-351, 1987.

[8] GREEN, B.G., FRANKMANN, S.P. The effect of cooling on the perceived intensity of taste. Chem. Senses, v.12, p. 609-19, 1987.

[9] ISIMA,N., KAKAYAMA,O. Sensory evaluation of stevioside as a sweetener. Shokuhin Sogo Kenkyusho Kenkyu Hokoku, v.31, p.80-5, 1976.

[10] LAWLESS, H.T. \& HEYMANN, H. Sensory evaluation of food. Gaythesburg, Maryland: Aspen Publ., 1999, $827 \mathrm{p}$.

[11] MEILGAard, M., CiVILle, G.V., CARR, B.T. Sensory evaluation techniques. $3^{\text {rd }}$. ed., New York: CRC Press, 1999, 387p

[12] MOSKOWITZ, H.R. Ratio scales of sugar sweetness. Percept. Psychophys, v.7, p.315-20, 1970.

[13] PORTMANn, M.O., SERGHAT, S., MATHLOUTHi, M. Study of some factors affecting intensity/time characteristics of sweetness. Food Chem., v. 44, p.83921 1992 .

[14] ROBBERS, J.E., SPEEDIE, M.K., TYLER, V.E. Pharmacognosy and Pharmacobiotechnology. Baltimore: Williams \& Wilkins. 1997, 372p.

[15] STONE, H., OLIVER, S.M. Measurement of the relative sweetness of selected sweeteners and sweetener mixtures. J.Food Sci., v.34, p.215-22, 1969.

[16] TUNAlEy, A., THOMSON, D.M.H., McEWAN, J.A. Determination of equi-sweet concentrations of nine sweeteners using a relative rating technique. Int. J. Food Sci. Technol., v. 22, p. 627-351 1987.

[17] VICKERS, Z. Sensory specific satiety in lemonade using a just right scale for sweetness. J. Sensory Study, V.3, n1, p.1-8, 1988.

[18] YAMAGUCHI, S., YOSHIKAWA, T., IKEDA, S., NINOMIYA, T. Studies on the taste of some sweet substances Part 1. Inter-relationships among then. Agric. Biol. Chem., v. 34, p.181-6, 1970.

\section{6 - AGRADECIMENTOS}

Os autores agradecem ao CNPq e à FAEP (Fundo de Amparo ao Estudante e à Pesquisa da Universidade Estadual de Campinas), pelo suporte concedido. 